three patients who gross extrauterine disease and in the aforementioned patient who had not surgery. Eleven (48\%) patients had adjuvant treatments, consisting in anthracycline-based chemotherapy $(n=4)$,gemcitabine-based chemotherapy $(n=2)$, mTOR inhibitors $(n=4)$ and hormonal treatment $(n=1)$. Median (range) follow-up as 23 (2, 99) months. Eleven (48\%) recurrences occurred with a mean (SD) progression free-survival of 14 (11) months. After a median (range) follow-up of 23 (2, 99) months, nine (39\%) patients died of disease. Residual tumor at surgery was the only factor correlating with the risk of developing recurrent disease $(p=0.023)$ and worse overall survival $(p=0.014)$. In our small series, stage of disease and adjuvant therapy administration had no impact on survival outcomes.

Conclusion* Uterine PEComa represents a rare and aggressive entity. Molecular/genomic profiling of the disease is necessary to predict response to treatment. Further collaborative investigations are warranted to assess the role of various prognostic factors and evaluate the most effective surgical and medical treatment modalities.

\section{LOW PREOPERATIVE SKELETAL MUSCLE DENSITY PREDICTS POSTOPERATIVE COMPLICATIONS AND FUNCTIONAL DECLINE IN OLDER WOMEN WITH OVARIAN CANCER}

\begin{abstract}
${ }^{1} \mathrm{~V}$ Van der Zanden, ${ }^{2} \mathrm{NJ}$ Van Soolingen, ${ }^{3} \mathrm{~A}$ Viddeleer, ${ }^{2} \mathrm{~J}$ Trum, ${ }^{2 ; 4} \mathrm{~F}$ Amant, ${ }^{5} \mathrm{MJE}$ Mourits*, ${ }^{6} \mathrm{~J}$ Portielje, ${ }^{6} \mathrm{~F}$ Van den Bos, ${ }^{7} \mathrm{C}$ De Kroon, ${ }^{8} \mathrm{M}$ Kagie, ${ }^{9} \mathrm{~S}$ Oei, ${ }^{10} \mathrm{~A}$ Baalbergen, ${ }^{11} \mathrm{AMLDVan}$ Haaften-de Jong, ${ }^{12} \mathrm{D}$ Houtsma, ${ }^{1} \mathrm{~B}$ Van Munster, ${ }^{6} \mathrm{E}$ Souwer. ${ }^{1}$ University Medical Centre Groningen, Internal Medicine, Groningen, Netherlands; ${ }^{2}$ The Netherlands Cancer Institute, Department of Gynaecologic Oncology, Amsterdam, Netherlands; ${ }^{3}$ University Medical Center Groningen, Medical Imaging Center, Department of Radiology, Groningen, Netherlands; ${ }^{4} \mathrm{KU}$ Leuven, Oncology, Leuven, Belgium; ${ }^{5}$ University Medical Centre Groningen, Gynecological Oncology, Groningen, Netherlands; ${ }^{6}$ Leiden University Medical Centre, Department of Medical Oncology, Leiden, Netherlands; 'Leiden University Medical Centre, Department of Obstetrics and Gynecology, Leiden, Netherlands; ${ }^{8}$ Haaglanden Medical Centre, Department of Obstetrics and Gynecology, The Hague, Netherlands; ${ }^{9}$ Haaglanden Medical Centre, Radiology, The Hague, Netherlands; ${ }^{10}$ Reinier de Graaf Group, Department of Obstetrics and Gynecology, Delft, Netherlands; ${ }^{11}$ Haga Medical Centre, Department of Obstetrics and Gynecology, The Hague, Netherlands; ${ }^{12}$ Haga Medical Centre, Department of Medical Oncology, The Hague, Netherlands
\end{abstract}

\subsection{6/ijgc-2021-ESGO.251}

Introduction/Background* Insights in how to select older patients who can benefit from standard care and patients that need adjusted treatment are necessary. This study aims to determine the predictive value of lumbar skeletal muscle mass and density, measured on a computed tomography (CT) scan, for postoperative outcomes in older women with advanced stage ovarian cancer.

Methodology A multicentre, retrospective cohort study was performed in women $\geq 70$ years old with advanced stage ovarian cancer who underwent surgery. Skeletal muscle mass and density were assessed in axial CT slices on level L3. Low skeletal muscle mass was defined as skeletal muscle index $<38.50$ $\mathrm{cm}^{2} / \mathrm{m}^{2}$. Low skeletal muscle density was defined as one standard deviation below the mean (muscle attenuation $<22.55$ Hounsfield Units). The primary outcome was any postoperative complication $\leq 30$ days after surgery. Secondary outcomes included severe complications, infections, delirium, prolonged hospital stay, discharge destination, discontinuation of adjuvant chemotherapy and mortality.

To investigate whether skeletal muscle density was of added value as a predictor for postoperative complications, we first
Abstract 142 Table 1 Results from univariable and multivariable analysis of predictors used to build the multivariable predictable model for postoperative complications within 30 Days after surgery

\begin{tabular}{|c|c|c|c|c|c|c|}
\hline Potential Predictors & $\begin{array}{l}\text { Odds } \\
\text { ratio }\end{array}$ & $\begin{array}{l}95 \% \\
\text { confidence } \\
\text { interval }\end{array}$ & $p$-value & $\begin{array}{l}\text { Odds } \\
\text { ratio }\end{array}$ & $\begin{array}{l}95 \% \\
\text { confidence } \\
\text { interval }\end{array}$ & $p$-value \\
\hline & \multicolumn{3}{|c|}{ Univariable analysis } & \multicolumn{3}{|c|}{ Multivariable analysis (n=178) } \\
\hline Low skeletal muscle density* & 2.83 & $1.41-5.67$ & 0.003 & 2.57 & $1.21-5.45$ & 0.01 \\
\hline KATZ-ADL $\geq 2(n=178)$ & 3.11 & $1.05-9.20$ & 0.04 & 2.67 & $0.88-8.12$ & 0.08 \\
\hline \multicolumn{7}{|c|}{ Candidate predictors associated with postoperative complications $(p<0.20)$, but not improving the model } \\
\hline Bowel surgery $(\mathbf{n}=\mathbf{2 1 0})$ & 1.92 & $0.90-4.12$ & 0.09 & & & \\
\hline FIGO stage IV & 1.61 & $0.87-2.99$ & 0.13 & & & \\
\hline Charlson Comorbidity Index $\geq 2$ & 0.61 & $0.32-1.18$ & 0.14 & & & \\
\hline Polypharmacy ${ }^{\dagger}$ & 1.55 & $0.85-2.82$ & 0.15 & & & \\
\hline \multicolumn{7}{|c|}{ Candidate predictors not associated with postoperative complications $(p \geq 0.20)$} \\
\hline Living at home with help ( $\mathbf{n}=\mathbf{2 1 0}$ ) & 1.62 & $0.72-3.65$ & 0.24 & & & \\
\hline Undifferentiated tumor ( $\mathrm{n}=195$ ) & 4.67 & $0.30-73.38$ & 0.27 & & & \\
\hline Use of walking aid ( $n=178$ ) & 1.52 & $0.66-3.49$ & 0.32 & & & \\
\hline Risk for malnutrition ( $\mathrm{n}=117$ ) & 1.42 & $0.65-3.08$ & 0.38 & & & \\
\hline ASA score $3 \neq(n=211)$ & 1.50 & $0.58-3.87$ & 0.40 & & & \\
\hline Body Mass Index & 1.03 & $0.96-1.09$ & 0.46 & & & \\
\hline History of confusion during illness ( $\mathrm{n}=\mathbf{1 8 1}$ ) & 1.43 & $0.31-6.58$ & 0.65 & & & \\
\hline Fall risk ( $n=173)$ & 1.21 & $0.51-2.87$ & 0.66 & & & \\
\hline Age & 0.99 & $0.92-1.06$ & 0.72 & & & \\
\hline Pre-existing memory problems ( $n=181$ ) & 0.79 & $0.20-3.18$ & 0.74 & & & \\
\hline \multicolumn{7}{|c|}{ 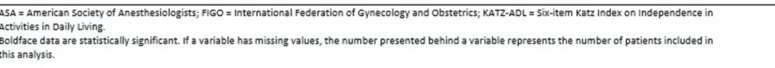 } \\
\hline 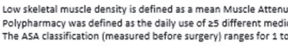 & $\begin{array}{l}\text { tion (MA) } \\
\text { tes with higt }\end{array}$ & u. & 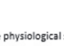 & 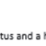 & risk. & \\
\hline
\end{tabular}

built a model with pre-existing relevant preoperative predictors only. After this model was built, we added skeletal muscle density to assess if it improved the model. A statistically significant step Chi-square statistic demonstrated that the new model performed better than the model with existing predictors.

Result(s)* 213 Patients were included. Preoperative low skeletal muscle density was associated with postoperative complications $\leq 30$ days after surgery (Odds Ratio (OR) 2.83; 95\% Confidence Interval (CI) 1.41-5.67), severe complications (OR 3.01; 95\%CI 1.09-8.33), infectious complications (OR 2.79; 95\% CI 1.30-5.99) and discharge to a care facility (OR 3.04; 95\%CI 1.16-7.93). Preoperative low skeletal muscle mass was only associated with infectious complications (OR 2.32;95\% CI 1.09-4.92). In a multivariable model (table 1), low skeletal muscle density was of added predictive value for postoperative complications to the strongest existing predictor functional impairment (KATZ-ADL $\geq 2$ ) (OR 2.57; 95\%CI 1.21-5.45; step Chi-Square statistic $\mathrm{p}=0.01)$.

Conclusion* Low skeletal muscle density, as a proxy of muscle quality, is associated with worse postoperative outcomes in older patients with advanced stage ovarian cancer. These findings can contribute to preoperative risk assessment and clinical decision making.

\section{USEFULNESS OF HYSTEROSCOPY IN THE MANAGEMENT OF BREAST CANCER PATIENTS WITH TAMOXIFEN AS AN ADJUVANT TREATMENT}

JM Barreiro García, M Marti Sopeña, S Álvarez Sánchez*, JJ Delgado Espeja, JA Solano Calvo, A Zapico Goñi. Prince of Asturias University Hospital, Obstetrics and Gynecology, Meco, Spain

10.1136/ijgc-2021-ESG0.252 
Introduction/Background* An increased risk of endometrial cancer and other endometrial pathologies is associated with the use of tamoxifen. Our objective is to evaluate the hysteroscopic approach on patients who had received tamoxifen as an adjuvant therapy for a breast cancer.

Methodology Retrospective observational study of 7406 office hysteroscopies performed between January 2010 and December 2019 at our university hospital. 213 patients who have received tamoxifen were found and included on this study.

Result(s)* The mean age was 53.6 \pm 10.1 years. 167 patients (78\%) were postmenopausal, while 45 (22\%) were premenopausal.

138 office hysteroscopies were performed on symptomatic patients: 128 patients (60\%) with postmenopausal bleeding and 10 patients (5\%) with hypermenorrhea. Besides, 66 asymptomatic patients (30\%) with abnormal ultrasound underwent an office hysteroscopy.

Ultrasound performed before hysteroscopy allowed to reveal 86 endometrial polyps (41\%), 10 submucosal myomas $(5 \%)$ and 11 patients with endometrial increased thickness (5\%). On the 105 patients left (50\%), ultrasound was normal.

17 office hysteroscopies could not be performed because of cervical stenosis. We found 88 (42\%) endometrial polyps, 7 (3\%) submucosal myomas and $3(2 \%)$ uterine malformations. $85(41 \%)$ patients underwent the hysteroscopy without abnormal findings. Hysteroscopy was suggestive of endometrial hyperplasia on 8 patients (4\%). Findings were highly suggestive of endometrial carcinoma on 4 patients $(2 \%)$.

On the histologic examination, malignancy was confirmed on the 4 patients with hysteroscopy suggestive of endometrial adenocarcinoma, while endometrial hyperplasia was confirmed on 13 patients (6\%).

Two of the four patients with diagnosis of endometrial adenocarcinoma were symptomatic (postmenopausal bleeding). Ultrasound on these patients showed endometrial polyps. The other two patients were performed a hysteroscopy because of increased endometrial thickness. Hysteroscopy revealed endometrial neoformations with atypical vascularization.

Conclusion* Our results show the increased risk of endometrial pathology on tamoxifen-treated patients, according to the existing literature. Ultrasound can be a good first approach on asymptomatic patients, but with limited accuracy. However, hysteroscopy is the best way of evaluating the endometrial cavity, making possible to see and treat endometrial pathologies on the same procedure. Furthermore, more studies are needed to validate the use of hysteroscopy as a first approach on symptomatic patients.

\section{PAZODOBLE - PAZOPANIB VS. PAZOPANIB PLUS GEMCITABINE IN RELAPSED OR METASTATIC UTERINE LEIOMYOSARCOMAS OR UTERINE CARCINOSACOMAS}

${ }^{1}$ LA Otten*, ${ }^{2}$ S Sehouli, ${ }^{3} E M$ Grischke, ${ }^{4} D$ Pink, ${ }^{5} P$ Reichardt, ${ }^{6} \mathrm{P}$ Harter, ${ }^{7} \mathrm{P}$ Wimberger, ${ }^{8} \mathrm{Z}$ Alwafai, ${ }^{9} \mathrm{BB}$ Runnebaum, ${ }^{1} \mathrm{~A}$ Mustea. ${ }^{1}$ Universitätsklinikum Bonn, Klinik für Gynäkologie und Gynäkologische Onkologie; ${ }^{2}$ Universitätsmedizin Berlin Charité Campus VirchowKlinikum; ${ }^{3}$ Universitätsfrauenklinik Tübingen; ${ }^{4}$ Helios Klinikum Bad Saarow, Hämatologie, Onkologie und Palliativmedizin, Sarkomzentrum; ${ }^{5}$ Helios Klinikum Berlin-Buch, Klinik für Onkologie und Paliativmedizin; ${ }^{6}$ Ev. KlinikenEssen-Mitte, Department of Gynecology and Gynecologic Oncology; ' Universitätsklinik Carl Gustav Carus der Technischen Universität Dresden, Klinik für Frauenheilkunde und Geburtshilfe; ${ }^{8}$ Universitätsmedizin Greifswald, Klinik und Poliklinik für Frauenheilkunde und Geburtshilfe; ${ }^{9}$ Universitätsklinikum Jena

\subsection{6/ijgc-2021-ESG0.253}

Introduction/Background" As there are only a few therapy options for advanced or recurrent leiomyosarcoma (LMS) and Carcinosarcomas (CS), new effective drug combinations with an acceptable toxicity profile are urgently needed.

Pazopanib is an orally available second-generation multi targeted tyrosine kinase inhibitor (TKI), targeting among others VEGFR, PFGG and c-kitand is approved in soft tissue sarcomas.

A phase I study evaluated the combination of pazopanib and gemcitabine and showed an acceptable toxicity profile. We aim to test the activity of the combination therapy in patients with LMS in a randomized setting and to describe the activity in CS.

Methodology The PazoDoble study is a prospective, randomized, open-label, multicentre phase II trial. Patients with relapsed or metastatic uterine LMS or uterine CS are eligible for participation. LMS are randomised in a $1: 1$ fashion into two treatment arms. A: pazopanib $800 \mathrm{mg}$ p.o./d plus gemcitabine $1000 \mathrm{mg} / \mathrm{m} 2$ i.v. over $30 \mathrm{~min} \mathrm{~d} 1$ und $\mathrm{d} 8 \mathrm{q} 3 \mathrm{w}$ or $\mathrm{B}$ : pazopanib $800 \mathrm{mg}$ p.o./d. Uterine CS will be enrolled only in Arm A. Duration of therapy will be maximum 18 months or until progression, death or unacceptable toxicity. Primary Endpoints are the six month PFS and clinical progression; Secondary endpoints are the objective response rate, duration of response, time to progression, OS, toxicity and tolerability and quality of life. A previousatment with doxorubicin or any contraindications against doxorubicin and an ECOG of 0 or 1 are mandatory. Clinical and imaging follow-up are scheduled every three month until disease progression or death.

Enrolment was started in October 2019 at nine sites. Until now, 12 Patients are enrolled (5 CS and 7 LMS). We aim to include 87 patients with LMS and 20 with CS. The primary endpoint will be analysed as the ratio of the number of patients showing no progress after 6 month divided by the
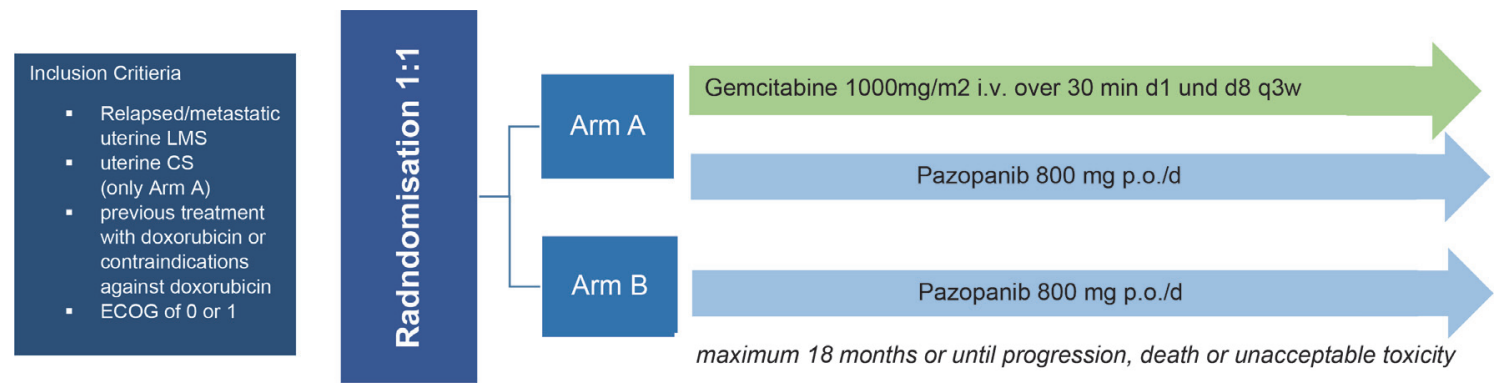

Abstract 169 Figure 1 